\title{
Using Wearable Sensors for Remote Healthcare Monitoring System
}

\author{
Ademola Philip Abidoye ${ }^{1,2}$, Nureni Ayofe Azeez ${ }^{1,3}$, Ademola Olusola Adesina ${ }^{1,2}$, Kehinde K. Agbele ${ }^{1,2}$, \\ Henry O. Nyongesa ${ }^{1,2}$ \\ ${ }^{1}$ Soft Computing and Natural Language Research Group, Bellville, South Africa \\ ${ }^{2}$ Department of Computer Science, Faculty of Natural Science, University of the Western Cape, \\ Bellville, South Africa \\ ${ }^{3}$ Grid Computing \& Distributed Systems Laboratory, Department of Computer Science, University of the Western Cape, \\ Bellville, South Africa \\ E-mail: \{ademola.abidoye, inadesina, henry.nyongesa\}@gmail.com,agbelek@yahoo.com,nurayhn@yahoo.ca \\ Received March 31, 2011; revised May 3, 2011; accepted May 22, 2011
}

\begin{abstract}
Recent technological advances in wireless communications and wireless sensor networks have enabled the design of low-cost, intelligent, tiny, and lightweight medical sensor nodes that can be strategically placed on human body, create a wireless body area network (WBAN) to monitor various physiological vital signs for a long period of time and providing real-time feedback to the user and medical staff. WBANs promise to revolutionize health monitoring. In this paper, medical sensors were used to collect physiological data from patients and transmit it to Intelligent Personal digital Assistant (IPDA) using ZigBee/IEEE802.15.4 standard and to medical server using $3 \mathrm{G}$ communications. We introduced priority scheduling and data compression into the system to increase transmission rate of physiological critical signals which improve the bandwidth utilization. It also extends the life time of hand-held personal server by reducing power consumption during transmission.
\end{abstract}

Keywords: Wireless Sensor Networks, Wireless Body Area Networks, ZigBee/IEEE 802.15.4, PDA, 3G, Medical Server

\section{Introduction}

Wireless Sensor Networks (WSNs) with intelligent sensor nodes are becoming significant enabling technology for wide range applications. Recent technology advances in integration and miniaturization of physical sensors, microprocessors, and radio interfaces on a single chip, have enabled a new generation of wireless sensor networks suitable for many applications [1]. For example, they can be used for emergency response, industrial automation, military surveillance, seismic, environmental monitoring, agricultural applications, and healthcare monitoring. One of the most promising uses of WSNs is healthcare monitoring [2].

A wireless sensor network consists of many nodes equipped with a sensing unit, memory, microcontroller (microprocessor), wireless communication interface and power source constituting of a multi-hop network, where nodes in the same vicinity can communicate with each other with routing responsibilities. Contrary to the traditional sensor networks that are carefully planned and deployed in the predetermined positions, WSNs can be deployed in an ad-hoc manner which make them robust, fault tolerance, and increase in spatial coverage.

They can greatly be used to monitor and track conditions of patients in both cities and rural areas using an intranet or internet thereby reducing the stress and strain of healthcare providers, eliminate medical errors, reduce workload and increase efficiency of hospital staff, reduce long-term cost of healthcare services, and improve the comfort of the patients [3].

These errors occur due to lack of correct and complete information at the location at the time it is needed, resulting in wrong diagnosis and drug interaction problems [4].

Mortality can be reduced if the patients are provided with correct instructions at the right time.

To ensure patients safety and save lives, it is necessary 
for medical staff to have access to patients' information within short period of time and at the right time.

Therefore, providing guaranteed, secured, and low transmission latency for patients vital signs are of great importance for life threatening diseases such as cardiovascular diseases, temperature, blood pressure etc.

Sensor nodes can be strategically placed on the human body create a cluster that is called wireless body area network (WBAN) that can be used to collect patient's vital signs [5].

It is worth noting that sensor nodes are being operated by batteries, their power consumption during transmission must be minimal for efficient and reliable data transmission between WBAN and personal server.

Using sensor nodes with communication technologies such as mobile phones i.e. PDA, General Packet Radio Service (GPRS), 3G, and the internet, the sensor network can keep patient, caregivers, and doctor informed while also establishing trends and detecting variations in health.

When they are applied to biomedical applications they are often referred to as wireless biomedical sensor networks (WBSN) [6].

WBSN allows the integration of low-power, miniaturized, intelligent pervasive sensor nodes to monitor body functions and the surrounding environment. Each node has capability to sense, process and forward the information to the Super sensor.

Stated below are some advantages of body sensor networks (BSNs).

Firstly, patients equipped with BSNs need not be physically present at the physician for their diagnostic.

Secondly, physicians can read patient's physiological data in time and then give real-time diagnosis advices which are important to patient's recovery.

Thirdly, unobtrusive, ambulatory wearable sensors allow physiological data to be collected automatically, reducing the transportation cost and regular visits to the physician [7].

Fourthly, a physician can take care of a few patients at the same time, thus reduces personnel expenses [8].

BSNs can detect small changes in vital signals e.g heart rate and blood-oxygen levels that are not obvious in a one-off visit to a doctor.

Finally, in case of emergency, BSN proves to be adequate, because it autonomously sends data about the patient health so that medical staff can prepare for the treatment immediately [9].

In this paper, we propose a networking solution in which a Medical Super Sensor (MSS) is used to collect multiple physiological signs sensed by each of the body sensors in WBAN and forward them to the personal server. An Intelligent Personal Digital Assistant (IPDA) is used as a personal server; it has the ability to collect patient's vital signs and prioritizes the data transmission based on patient's current condition and data content.

The rest of the paper is organized as follows. Section 2 explains some applications of wireless sensors, Section 3 presents the system architecture and the three tiers of the system are explained finally, conclusion is presented in Section 4.

\section{Healthcare Applications of Wireless Sensors}

\section{Some of the Applications of Wireless Sensors in Heal- thcare are Stated Below.}

1) Heart Diseases

Cardiovascular disease refers to various medical conditions that affect the heart and blood vessels. The conditions include heart attack, heart failure, stroke, coronary artery disease. This disease is the leading cause of mortality in the developed world $[10,11]$.

World Heart Organization [12] stated that heart disease accounts for about 17 million (about 30\%) deaths annually throughout the world and 80 percent of all deaths in China are caused by chronic disease [13] About one-half of those who die do so within 1 hour of the start of symptoms and before reaching the hospital.

A WBAN is a key technology that provides real-time monitoring of cardiovascular patients by continuously sense, process, and transmit physiological data from central control unit to the medical server through personal server, which the physician can make use of the information to treat the patients. Also promoting timely intervention of health care structure as and when required.

\section{2) Asthma}

Millions of patients are suffering from asthma in the world, a WBAN can help these patients by monitoring allergic agents in the air and providing real-time feedback to the physician and/or to the patient himself. A portable Global Positioning System (GPS) device was developed by Chu et al. [14], that continuously consults a remote server by sensing user's reports to decide whether current ambient air quality will threaten user's health. The server also collects real-time data from the network of national air quality monitoring stations. If it finds anything allergic to the patient, an alarm to the patient and/or physician will be triggered.

\section{3) Cancer Detection}

National Centre for Health Statistics in their annual report stated that about 9 million cancer patients were diagnosed in 1999 and the number is increasing every year [15]. Cancer is now one of the biggest threats to the human life. A WBAN with a set of miniaturized sensors can be used to differentiate between different types of 
cells and identifying cancerous cells, enabling physicians to diagnose tumors without biopsy.

4) Diabetes

World Health Organization (WHO) reported that more that 220 million people worldwide have diabetes and 1.1 million people died from diabetes in 2005 . Following are some of the complications that occurs as a result of diabetes: amputations, blindness, kidney disease, stroke, high blood pressure, heart disease [16] Treatment includes blood pressure control, exercise, insulin injections. A WBAN can be used in a more effective way to treat diabetes, by providing a more consistent, less invasive and accurate method for monitoring glucose levels in the body [17].

\section{5) Artificial Retina}

Optoelectronic Retina Prosthesis (ORP) chips can be implanted into the back of human eye, which assist blinds and/or patients with low vision to see normally. More information on www.mdsupport.org.

\section{System Architecture}

This section describes the system architecture of the proposed wearable sensors for remote healthcare monitoring system. The system is composed of three tiers as shown in Figure 1 below.

The system composed of:

1) Wireless Body Area Network (WBAN);

2) Personal Server (PPS) using IPDA;

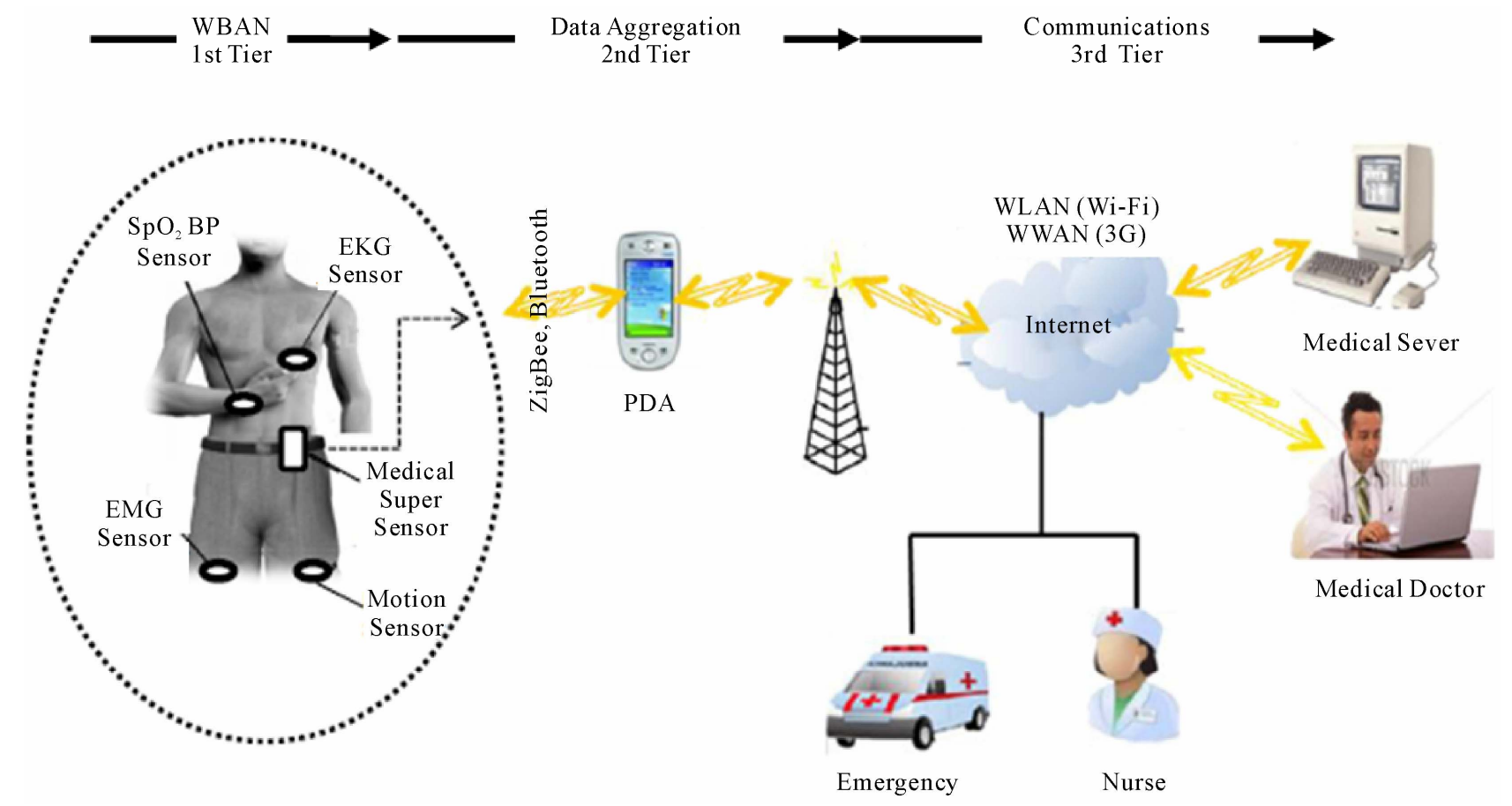

3) Medical Server for Healthcare Monitoring (MSHM).

\subsection{First Tier}

The core of this system is the user called the patient. Wearable sensors are attacked to the patient body forming wireless body area network (WBAN) to monitor changes in patient's vital signs closely and provide real time feedback to help maintain an optimal health status. The medical sensors typically consist of five main components:

1) Sensor: it is a sensing chip to sense physiological data from the patient's body.

2) Microcontroller: it is used to perform local data processing such as data compression and it also controls the functionality of other components in the sensor node.

3) Memory: it is used to store sensed data temporally.

4) Radio Transceiver: it is responsible for communication between nodes and to send/receive sensed physiological data wirelessly.

5) Power supply: the sensor nodes are powered by batteries with a lifetime of several months [18].

Sensor nodes can sense, sample, and process one or more physiological signals. For example, an electrocardiography (EKG) sensor can be used for monitoring heart activity, a blood pressure sensor can be used for monitoring blood pressure, a breathing sensor for monitoring respiration, an electromyogram (EMG) sensor for monitoring muscle activity, and an electroenpha-

Figure 1. Architecture of wearable sensors for remote healthcare monitoring system [4]. 
logram (EEG) sensor for monitoring brain electrical arrive for each sensor.

In our design, a sophisticated sensor is integrated into the WBAN called Medical Super Sensor (MSS). This sensor has more memory, processing and communication capabilities than other sensor nodes as shown in Figure 1 above. MSS uses a radio frequency to communicate with other body sensors and ZigBee is used as a communication protocol to communicate with the Personal Server.

In this design, we considered Bluetooth and ZigBee technologies. In case of Bluetooth specification, it supports maximum of seven active slaves (i.e. sensors to be controlled by one master, personal server). But the number of sensor nodes we are considering in this system are more than seven therefore Bluetooth technology is not acceptable option.

The second technology is ZigBee/IEEE 802.15.4 standard. It has a short range, low power consumption, low cost technology, capable of handling large sensor networks up to 65,000 nodes and reliable data transfer. It supports a maximum of $250 \mathrm{kbps}$ using Industrial, Scientific and Medical (ISM) free band i.e. 2.4 GHz. Therefore, $\mathrm{ZigBee}$ is adopted to transmit physiological signals from WBAN to the patient server.

Other reasons why it is used are stated below:

- Security: Patient information is vital; it must not be changed by unauthorized person. Data transfers from WBAN to the personal server and the medical server must be secured. ZigBee provides a low power hardware encryption solution using Advanced Encryption Standard (AES 128) to encrypt data transmitted between MSS and personal server.

- Scalability: it is highly scalable for many devices.

- Interoperability between a variety of medical and non-medical devices with data management devices regardless of manufacturer.

However, Medical Super-Sensor (MSS) unobtrusively samples, collects multiple sensed vital signs by the body sensors, filtering out all redundant data thereby reducing large volume of data transmitted by BSNs, store them temporarily, process and transfer the relevant patient's data to a personal server through wireless personal implemented using ZigBee/IEEE 802.15.4. This improves overall bandwidth utilization as well as reducing power consumption of the BSs because each nodes does not need to transmit sensed data to the IPDA but to the collector which is MSS and it is closer to the BSs than IPDA and extending battery life of each sensor node.

\subsection{Second Tier}

\section{Personal Server}

The personal server interfaces the WBAN nodes through a communication protocol using ZigBee. It is implemented on an Intelligent Personal Digital Assistant (IPDA). It holds patient authentication information and is configured with the medical server IP address in order to interface the medical services. It collects physiological vital signals from WBAN, processes them, and prioritizes the transmission of critical data when there is sudden clinical change in the current patient condition and data content for example changes in cardiovascular signals, temperature, oxygen saturation, and forward it to the medical server.

Moreover, the IPDA has the capability to perform the task of analyzing the physiological data intelligently and do a local reasoning to determine user's health status based on data received from MSS and provide feedback through a user-friendly and interactive graphical user interface. 3G communications is used to connect personal server and third tier together but other long range communications protocols can also be used like GPRS, WWAN.

In order for IPDA to improve the overall quality of

Table 1. Physiological signal characteristics.

\begin{tabular}{|c|c|c|c|}
\hline Physiological Signal & Parameter Range & Data Rate(Kbps) & Data Arrival Time (Sec) \\
\hline Electrocardiograph (EKG) & $0.5-4 \mathrm{mv}$ & 6.0 & 0.002 \\
\hline Blood Flow & $1-300 \mathrm{ml} / \mathrm{s}$ & 0.48 & 0.025 \\
\hline Respiratory Rate & $2-50$ breaths $/ \mathrm{min}$ & 0.24 & 0.05 \\
\hline Oxygen Saturation $\left(\mathrm{SpO}_{2}\right)$ & $0.01-0.85 / \mathrm{s}$ & 2.3 & 0.16 \\
\hline Blood Pressure & $10-400 \mathrm{mmHg}$ & 1.2 & 0.01 \\
\hline Blood PH & $6.8-7.8 \mathrm{PH}$ units & 0.048 & 0.25 \\
\hline Never Potentials & $0.01-3 \mathrm{mv}$ & 240 & $5 \mathrm{E}-05$ \\
\hline Body Temperature & $32-40^{\circ}$ & 0.0024 & 5 \\
\hline
\end{tabular}


service for data transmission, in terms of latency, bandwidth and power consumption a differentiated service based on two schemes are presented. They are Priority Scheduling and Data Compression.

\section{Priority Scheduling and Data Compression}

There are different physiological signals that are normally transmitted between the sensor nodes and patient server. The transmission is divided into four types according to their data rate and latency. They are classified as follow.

- High data rate and low latency traffic

- Low data rate and low latency traffic

- Low data rate and high latency traffic

- High data rate and high latency traffic

High data rate means critical signs that need to be transfer very fast with high reliability while low latency means time delay to the response of transmission of critical signals and should be as much as possible be short.

From Table 2 below, each of the physiological signs is given priority weight. It shows the order in which the physiological signs will transmit from IPDA to the medical server via $3 \mathrm{G}$ communications for further analysis and diagnosis by the medical staff.

Physiological sign that is allocated with priority 1 has the highest priority over other data and will be allowed to transmit first and the vital sign should be transmitted without any delay. It means the present condition of the patient is critical and he/she needs immediate attention of the medical staff while physiological sign with high data rate and high latency means the signal is not critical. It will compress according to a given ratio and stored in the local memory of the IPDA for later transmission after physiological signs with higher priority i.e. priority 1 , priority 2 have been allowed to transmit first.

From what has been discussed above, timely access to the patient's medical information is important for providing correct treatment and improving the overall safety of the patient's care. Priority scheduling method not only reduces the transmission delay for critical physiological signals, but also decreases traffic congestion. The total number of data sent reduced through data compression method. Therefore, the bandwidth utilization is improved thus, reduces total transmission time. However, IPDA is

Table 2. Priority of physiological signs.

\begin{tabular}{cccc}
\hline Physiological Signs & Data Rate & Latency & Priority \\
\hline $\begin{array}{c}\text { Electrocardiograph (EKG) } \\
\text { Heart Rate, Blood Flow, } \\
\text { Oxygen Saturation }\end{array}$ & High & Low & 1 \\
$\begin{array}{c}\text { Respiratory Rate, Blood } \\
\text { Pressure, Body Temperature } \\
\text { Never Potentials }\end{array}$ & Low & Low & 2 \\
\hline
\end{tabular}

using battery for its operation and high amount of energy is consumed during transmission [19].

This method reduces energy consumed by the IPDA during transmission since only the critical vital signs will transmit first while less critical signs are stored and transmit later.

Figure 2 below is the flowchart that shows the IPDA working modes. The modes consist of Active Mode and Inactive Mode.

IPDA is inactive mode when it has no data to receive from MSS or send to the medical server in order to save energy but wake up immediately from inactive to active mode to receive transmitted data and store it. It prioritizes all the received physiological data and send to the medical server based on the priority order so that the medical staff will be adequately prepared before the patient gets to them or send ambulance immediately to pick the patient so as to save his/her life.

Medical Server for Healthcare Monitoring (MSHM).

The third tier is called Medical Server for Healthcare Monitoring (MSHM). It receives data from the personal server, is the backbone of the entire architecture. It is situated at medical centers where medical services are provided. It is intelligent because it is capable of learning patient specific thresholds and learns from previous treatment records of a patient [20]. MSHM keeps electronic medical records (EMRs) of registered patients, which are accessible by different medical staff, including general practitioners, specialists and doctors from their offices in the hospital over the internet. The present state of the patient can be observed by the medical staff. MSHM is responsible for user authentication, accepting data from personal server, format and insert the received data into corresponding EMRs, analyze the data patterns.

The patient's physician can access the data and its patterns from his/her office via the intranet/internet and examine it to ensure the patient is within expected health metrics. If the received data is out of range (i.e. deviation from threshold) or recognize serious health anomalies condition, medical staff in the emergency unit can be notified to take necessary actions.

However, if the patient is in the remote area, the specialist doctor will observe the physiological data of the patient diagnose it, prescribe the necessary treatment and drugs for the patient. This information will sent back to the doctor in the remote hospital via the internet.

The MSHM also provides feedback instructions to the patient, such as physician's prescribed exercises.

\section{Conclusions}

In this paper, we described the architecture of wearable sensors for remote healthcare monitoring system which 


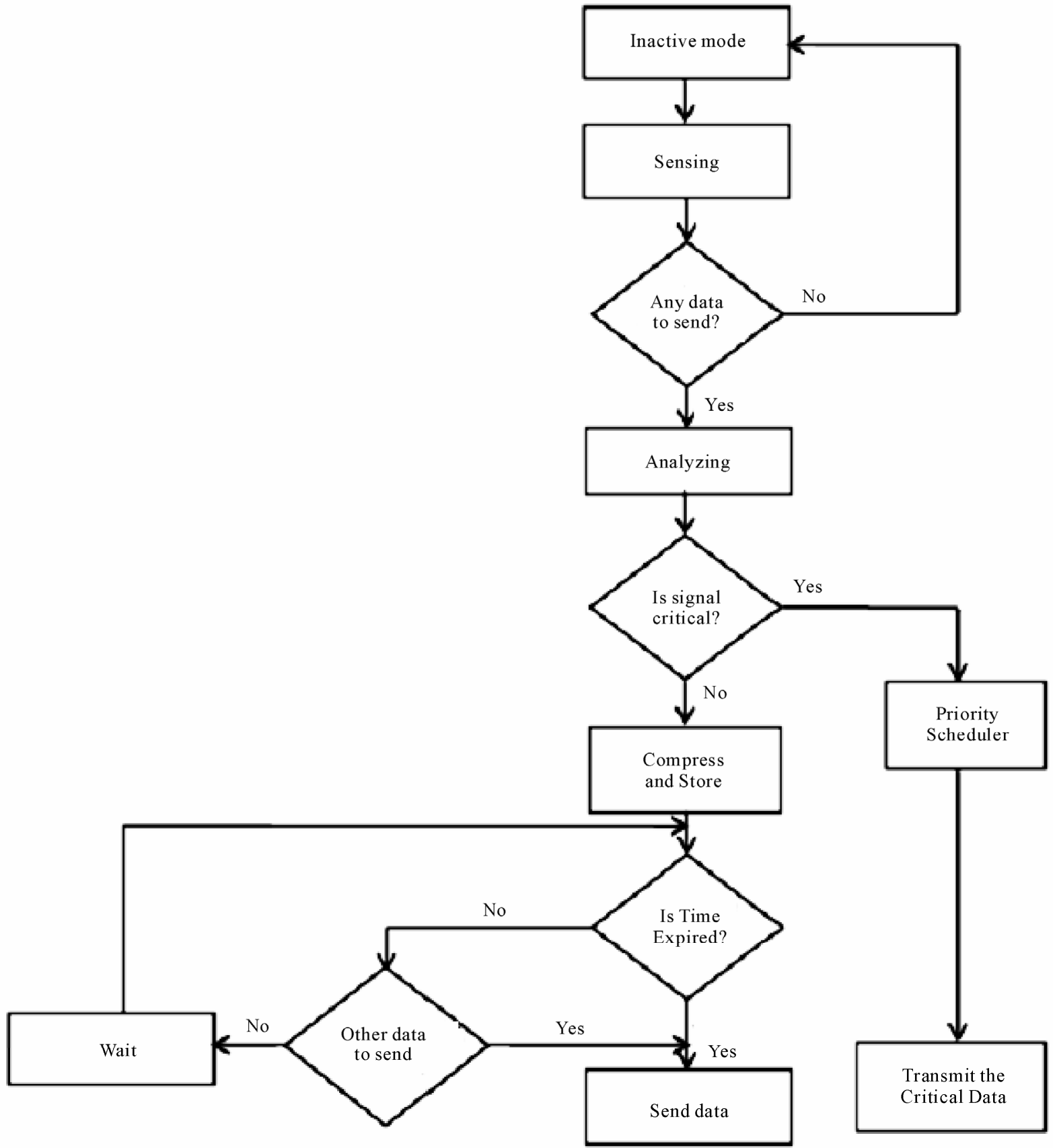

Figure 2. Flowchart of personal server working modes [8].

composed of three tiers. A differentiated services scheme based on priority scheduling and data compression methods were presented in second tier. The method not only reduces transmission delay of physiological vital signs but also improves its bandwidth utilization. The role of wireless technology in healthcare applications is expected to become more important with an increase in deployment of mobile devices and wireless networks. This new technology has potential to provide many advantages to patients, medical staff, and society at large through continuous monitoring of various physiological vital signs and provide real-time feedback to the user and the medical staff.

\section{References}

[1] W. Wang, et al., "CEDCAP: Cluster-Based Energy-Efficient Data Collecting and Aggregation Protocol for WSNs," Research Journal of Information Technology, Vol. 3, No. 2, 2011, pp. 93-103. 
[2] I. Khemapech, et al., "A Survey of Wireless Sensor Networks Technology," 6th Annual Postgraduate Symposium on the Convergence of Telecommunications, Networking and Broadcasting, Liverpool John Moores University, Liverpool, 2005.

[3] U. Varshney, "Pervasive Healthcare: Applications, Challenges and Wireless Solutions," Communications of the Association for Information Systems, Vol. 16, No. 1, 2005 , p. 3.

[4] V. Jones, et al., "Biosignal and Context Monitoring: Distributed Multimedia Applications of Body Area Networks in Healthcare," 2008 IEEE 10th Workshop on Multimedia Signal Processing, Cairns, 8-10 October 2008, pp. 820825.

[5] S. Vijendra, "Efficient Clustering for High Dimensional Data: Subspace Based Clustering and Density Based Clustering," Information Technology Journal, Vol. 10, No. 6, 2011, pp. 1092-1105. doi:10.3923/itj.2011.1092.1105

[6] X. Chen, et al., "Design of Sensor Node Platform for Wireless Biomedical Sensor Networks," 27th Annual International Conference of the Engineering in Medicine and Biology Society, Shanghai, 17-18 January 2006, pp. 4662-4665.

[7] A. Wood, et al., "ALARM-NET: Wireless Sensor Networks for Assisted-Living and Residential Monitoring," Technical Report CS-2006-13, University of Virginia, 2006.

[8] H. M. She, et al., "A Network-Based System Architecture for Remote Medical Applications," 2007. http://www.powershow.com

[9] E. Jovanov, et al., "A Wireless Body Area Network of Intelligent Motion Sensors for Computer Assisted Physical Rehabilitation," Journal of Neuroengineering and Rehabilitation, Vol. 2, No. 1, 2005, p. 6. doi:10.1186/1743-0003-2-6

[10] W. Rosamond, et al., "Heart Disease and Stroke Statistics-2007 Update: A Report from the American Heart Association Statistics Committee and Stroke Statistics
Subcommittee," Circulation, Vol. 115, No. 5, 2007, pp. e69-e171. doi:10.1161/CIRCULATIONAHA.106.179918

[11] I. Romero, et al., "Robust Beat Detector for Ambulatory Cardiac Monitoring," Annual International Conference on Engineering in Medicine and Biology Society, Minneapolis, 3-6 September 2009, pp. 950-953.

[12] World Health Organization, 2008. www.who.int

[13] RAND Corporation, "Home Health Care Could Help Sustain Health Care Systems, Study Finds," Trends in Applied Sciences Research, Vol. 6, No. 8, 2011, pp. 925-926.

[14] H. T. Chu, et al., "A Ubiquitous Warning System for Asthma-Inducement," IEEE International Conference on Sensor Networks, Ubiquitous, and Trustworthy Computing, Taichung, 5-7 June 2006, pp. 186-191.

[15] A. Milenkovic, et al., "Wireless Sensor Networks for Personal Health Monitoring: Issues and an Implementation," Computer Communications, Vol. 29, No. 13-14, 2006, pp. 2521-2533. doi:10.1016/j.comcom.2006.02.011

[16] A. A. Osman, "Management of Infertility within Primary Health Care Program in Sudan," Asian Journal of Scientific Research, Vol. 4, No. 2, 2011, pp. 158-164.

[17] Y. J. Zhao, et al., "A MEMS Viscometric Glucose Monitoring Device," The 13th IEE International Conference on Solid-State Sensors, Actuators and Microsystems, Pittsburgh, 5-9 June 2005, pp. 1816-1819.

[18] B. Lo and G. Z. Yang, "Key Technical Challenges and Current Implementations of Body Sensor Networks," 2005. http://ubimon.doc.ic.ac.uk/bsn/public/bsn-2005-Benlo.pdf

[19] G. J. Pottie and W. J. Kai, "Wireless Integrated Network Sensors," Communications of the ACM, Vol. 43, No. 5, 2000, pp. 51-58 . doi:10.1145/332833.332838

[20] M. Seyyed, et al., "Fuzzy Logic Expert Systems in Hospital: A Foundation View," Journal of Applied Sciences, Vol. 11, No. 12, 2011, pp. 2106-2110. 